\title{
Establishing a Pre and Post-3D Bodyscanning Survey Process for Able-Bodied UK Women Aged 55 Years+ to Determine an Appropriate Waist Position for Garment Development
}

\author{
Paula WREN ${ }^{* 1}$,Simeon GILL $^{2}$, Steven HAYES ${ }^{1}$, Phoebe APEAGYEI ${ }^{1}$ \\ ${ }^{1}$ Manchester Metropolitan University, Department of Apparel, Hollings Faculty, Manchester, UK; \\ ${ }^{2}$ Manchester University, School of Materials, Manchester, UK
}

http://dx.doi.org/10.15221/14.143

\begin{abstract}
Change in human body morphology is well-documented for developmental growth from childhood to adult. However, the morphological change from adulthood to older age is less detailed and less discussed within literature concerned with anthropometric application and clothing construction. This, perhaps, explains why females aged $55+$ are the demographic most frustrated with the fit of ready-to-wear garments [1]. A prerequisite for the accurate generation of size chart and pattern development relies on precision in the location and demarcation of landmarks [2,3], therefore an understanding of the changes in body size and shape, how this impacts on anthropometric practice, and, more specifically, the body landmarks is fundamental to provide correctly fitting garments patterns for mature women. Consequently, the aim of this research is to establish a pre- and post-3D body scanning survey process for able-bodied UK women aged 55 years and over, giving them the opportunity to self-select a suitable waist location for clothing from four defined waist positions.
\end{abstract}

A pragmatic, mixed-method methodology was developed to evaluate anthropometric theory and praxis, landmark placement, and subjective body image estimation, to determine the success of their practical application in developing appropriate garment patterns for this demographic.

The methodology consisted of four stages: recruitment of eighty (80) women aged 55+ using the three strategies of convenience, snowball and random sampling; the 3D body scanning of each participant using two TC[2] scanners; the use of a visual aid to allow the self-evaluation of personal waist position for garment development; and the evaluation of the visual and numerical scan data against this self-evaluation.

The findings indicated that recruitment of this demographic is problematic as it is reliant on subject willingness for participation and this group of subjects were often critical of their body morphology. The questionnaire response rate was $67 \%$ which reduced the sample size down to 52 women. The visual aid indicated that the participants were able to readily identify the position of their waist regardless of body morphology.

Rectangle, hourglass, triangle and bottom hourglass body shapes were represented in the sample set. The most common body shape shared by this demographic was that of the rectangle and analysis of waist height from the floor position before and after waist landmark modification indicated that the rectangle was the body shape which was most prone to waist placement error from the participants point of view. All body shapes required waist height modification to the original waist height landmark based on participant evaluation of where they felt it comfortable to wear their waistband. Comparative analysis confirmed distinct variation in subjects' evaluation of waist positioning for a garment and scanner MMU MEP definitions. 59\% of participants' waists landmarks were placed differently by the scanner compared to the placement by the subjects themselves.

This study concludes that to improve landmark placement accuracy providing a visual aid to the participant for evaluation in tandem with the practitioner evaluation would be a practice that is useful for common but difficult to locate landmarks, such as the waist. Responses from this visual aid operator evaluation the scan data and more specifically the landmarks.

Key words: Gerontology, 3D Body Scanning, Sizing Survey, Product Development, Anthropometrics

*P.Wren@mmu.ac.uk, +44 1612473552 


\section{Introduction}

As humans age, gradual degenerative body changes occur which can affect body size, shape and posture $[4,5]$. These changes affect garment fit and comfort levels as the body moves away from standard size and shape. Croney [4] lists these changes but focuses on child-to-adult morphological change, whereas information and schematics on adult-to-older age morphological change is less explicated. As body surface measurement data is necessary for the development of garment patterns that accurately fit the body [6,7], a greater understanding of body surface changes is necessary to enable practitioners to accurately extract the right measurements from the right areas of the body for subjects who no longer display a standard body size and shape. This knowledge would enable the development of block patterns that would provide both comfort and a pleasing aesthetic for this demographic.

Gross anatomical knowledge does not appear to be widespread in modern clothing measurement literature, with only Beazley and Bond [2], Kunick [8] and Bunka [9] offering some detail in this area. They all provide a level of detailed guidance on the acquisition of body surface measurements to enable the development of their block patterns but this comes in the form of manual measurements, which present challenges when applied to non-contact measurement technologies. These works provide visuals of the human form and a list of landmark definitions, which refer to gross anatomical reference points on the body from which skin surface measurements are taken [10]. However, Beazley and Bond [2], Kunick [8] and Bunka [9] use visuals and measurement definitions for standard sized female subjects who do not display body deviations away from the standard. Generating block patterns for the older female using this measurement guidance would present challenges.

\subsection{Morphological change and measurement systems}

Adult-to-older age morphological change can affect three phases of anthropometric measurement: how the landmarks are found and positioned; how measurements are taken; and manageability of posture adoption for certain measurements.

\subsubsection{Height and postural change}

Height is one morphological change linked to the ageing process, which impacts on all of the above three phases. Sorkin et al 's [11] study of height change found that height loss is greater in women than men. Lindsey et al's [12] study of the effects of hormone replacement therapy on 100 menopausal women correlated height loss with reduced hormone levels due to the onset of the menopause. This study found that subjects who had lower levels of bone density - a result of the menopausal transition displayed a significant reduction in height [12]. This can also lead to changes in upper body posture resulting in significant angle differences around the shoulder region compared to younger women [13]. Ashdown and $\mathrm{Na}$ [13] have explored and quantified upper postural body variation in both young (19-35) and mature (55+) female subjects using 3D-bodyscanning technology. This research found significant measurement angle differences around the upper torso between sample sets, highlighting that because the shoulders rolled forward the cervical portion of the back appeared more hunched. The findings of this study conceded that locating landmarks on the mature sample was difficult as the body changes affected areas where the landmarks are located on a younger person.

\subsubsection{Fat deposition}

Ley et al's [14] study of pre- and post-menopausal women found that post-menopausal participants typically have a higher body mass index (BMI) than pre-menopausal women, and fat distribution tended to be concentrated at the lower torso region around the abdomen. This finding concurs with previous studies wherein mature females had larger waist and abdominal arcs $[15,5,16,17,18]$. A study of Korean women's body shapes by Lee et al [19] found change also occurred in the bust and hip girths with a thickening of the waist region and an increasingly extended abdomen, something which is common regardless of ethnicity. Furthermore, although it is possible to retain fat deposits around the abdomen region regardless of age, a UK study undertaken by Wells et al [20] exploring age-associated body shape changes in young and mature women found that both women in their 20 s and over 55 years with the same BMI displayed different body shapes, dependent on their age. Younger obese females retained an indentation in the waist-area whereas mature females displayed higher android fat distribution around the abdomen and waist, thereby having less indentation in the waist region. This finding aligns with earlier [17] surveys which established that females over the age of 55 were most likely to display a rectangle torso body shape, which can be defined as where bust and hips circumferences are fairly equal and bust-to-waist and hip-to-waist ratios are low [19]. 


\subsubsection{Non-contact waist definitions}

The 3D body scanner works with the topography of the skin generating meshes, consisting of over 100,000 co-ordinate points, to define a human shape [21]. As the scanner is a non-contact measurement technology it 'sees' the surface points and traces pits and elevations in the topography of the skin surface to find specific regions for landmark placement. Therefore, definitions that require manual palpation are redundant for this technology. The TC[2] scanner locates the region of the waist using the spinal curve [22] instead of manual palpation to locate the mid region between the bottom rib and the top of the iliac crest (ISO 8559) which is an international standard manual measurement waist definition. Previous studies have indicated a need for greater landmark definitions for the scanner software to improve the accuracy of measurement placement [23] and this is particularly important for mature women who may experience the lowest rib lying against the top of the iliac crest - known as costo-iliac impingement syndrome - because of an osteoporotic condition [24]. This condition makes the ISO 8559 redundant, as clinically the individual has no longer a gap between the bottom rib and the iliac crest.

\subsection{Demographical attitudes regarding body scanning}

There have been a number of anthropometric surveys with the potential to collect measurement data from women aged 55+ $[25,15,16,17]$. Many of these surveys report encountering reluctance on the part of the subject to be seen in a state of semi-undress and had to provide specially made garments to protect the modesty of this demographic or did not recruit comparable numbers to the younger demographic sample in these surveys. The introduction of 3D body scanning can counter issues of modesty. The Lee et al [19] study investigating women's attitudes towards the scanning process indicated that $64 \%$ of older women were open to the thought of having their body scanned for the purpose of pattern development to improve garment fit. However, the study also acknowledged that when questioned whether they would be comfortable showing their scanned body image to friends or family, women who weighed more or who had a high BMI score would be uncomfortable letting others view their scan. This study indicates theoretical openness towards body scanning. However, as this sample group was not actually scanned these results could have been different had participants been required to be scanned.

\subsection{Self-evaluation of personal waist position and 3D body scanning}

\subsubsection{Validating self -evaluated waist measurements}

Disparate fields of study have explored self-reported body measurement to determine their reliability and validity. Rim et al's [27] early anthropometric study of women aged 41-65 years study found that participant's self-evaluated measurement of the waist using manual measurement protocols was very close to the manual measurements recorded by a technician, with no over or underestimation of waist circumference. Guerra et al's [28] more recent examination of anatomical location for waist circumference using manual measurement on a sample of 51 adults aged $60-85$, remarked that anatomical land marking is problematic in older subjects whose body morphology displayed excess abdominal visceral fat around the torso. Their work indicates that the only way to find the waist on an older female demographic was to palpate the participant's body surface using the skeletal-muscular framework alongside easily identifiable body surface attributes as landmarks. The work confirms that engaging with the participant in this way also provided the opportunity for verbal confirmation from the subject that the practitioner had marked out a landmark that correctly aligned with the subject's perception of where their own waist was.

Song \& Ashdown's [29] recent US study assessed the reliability of participant self-evaluated lower-body size and shape using 3D-body scanning technology in conjunction with the SizeUSA survey measurement definitions which places the waist landmark directly on the small of the back. The study compared the scanned body shape against the shape participants believed themselves to be. Importantly, the findings revealed a discrepancy between the self-evaluated waist height dimension and the placement of the waist height by the body scanner with respondents indicating their waist height should be shorter in their view. Furthermore, those reporting most discrepancy between self-evaluation and the scanner were those whose body displayed a fuller silhouette. This does indicate the waist height definition used by the scanner survey differs from where some participants understand their waist position to be, with most variance in subjects who have a fuller figure. Data from national surveys such as SizeUSA has been used for a number of industries including garment product development, so if the waist definition does not correspond to where members of the population who have a fuller silhouette understand their waist to be, garment producers may find issue with the waist on garments developed using this data. 


\subsection{Quantitative and qualitative methods to improve measurement protocols}

Haffenden's [30] study provides more targeted insights into the perceptions of older females whose body morphology displays a less defined waist position in her study centered on product development for knitted garments. This study employed mixed method approach using online questionnaires to gather broad opinions of garment fit before moving on to semi-structured interviews and a measurement survey using 3D scanning technology. The work collected key dimensions for garment development and established that the waist was a contentious area because large women do not conform to standard sizing. It also identified that participants were knowledgeable regarding their own body shape and were able to articulate their shape clearly using visual analysis of their body surface contours as well as proportional linear measurements. This work [30] is significant as it acknowledges that new protocols are needed when devising a measurement system for women who do not have a clearly defined waist.

\subsection{Mature women's requirements and knowledge of garment fit}

Recent studies surrounding mature clothing preference $[31,32,33]$ indicate this demographic desires clothing which not only looks appealing and is pertinent to their lifestyles but that is developed to correctly fit their body size and shape. Correct garment fit, is the most important aspect when considering a garments purchase [34,35], with perceptions on fit differing from one individual to another [23]. Research regarding preferences for aesthetic attributes in clothing as a function of both comfort and how individuals view their body image is ongoing. Richard's [36] early research found women over 65 years understood their body's physical attributes and had definite ideas on how clothing can be perceived as a corrective medium to balance out or obscure changes in body morphology. It has been established that this demographic is interested in particular garment styling and pattern amendment because of the changes in their body's morphology $[19,17,36]$ or examined anthropometric data to help amend pattern blocks [16].

Interpreting scan data into 2D pattern shapes is already under investigation [37]. However, no current research has solicited ideas on landmark positioning both pre and post-body scanning for the implementation into the pattern, which is central to this study.

\section{Methodology}

\subsection{Recruitment}

\subsubsection{Sampling strategies}

Recruitment of the females participants aged 55+ followed four different approaches: (1) posters were used to advertise the survey placing them in in public areas such as libraries, health clubs and doctors surgeries all situated within the Greater Manchester (UK) environs. (2) a convenience sample of individuals within the Manchester Metropolitan University (MMU) population were emailed. (3) snowball sampling via word-of-mouth contacts. (4) a random sample using the internet and local radio to a three-day scanning event held in Nottingham, UK.

Samples from recruitment approaches (1) to (3) were conveniently accessible to the study and selected using a non-probability sampling technique $[38,39]$. In addition to being female and $55+$ participants needed to be physically able to stand within the scanning booth for the time it took to capture their body surface data. No restriction was placed on height, body shape or size. The final sample number for scanning was 80 . The participant criteria - female, $55+$, physically able to stand was adhered to for recruitment approach (4) but this was deemed a random sample rather than a convenience sample due to the communication medium used to recruit. This type of mixed sampling approach added confidence to the findings [40], as it offered the opportunity to identify if a finding is affected by the chosen method of sampling.

\subsubsection{Final sample total}

80 participants were recruited and scanned. This sample number reduced as only 52 of the 80 scanned also participated in the use of the visual aid that constituted a part of the methodology of this study. The BS EN ISO 20685:2010 [41] standard recommends a sample size of 40 participants '[as] this will ensure $95 \%$ confidence in the validation test results for large circumferences such as chest, waist, and hip, which are particularly difficult to measure for both traditional and 3-D measurement systems'. As this study used a sample of 52 , an increase on the recommended 40 , it could be argued that this study justifies a high level of confidence in the validation of its own results . 


\subsubsection{Data types for mixed methods}

Sampling quotas for studies using mixed methods must include a strand that collects 'persuasive' qualitative data collection procedures, and a strand that incorporates rigorous quantitative procedures [42]. This study complies with this approach by gathering qualitative data concerning the thoughts of the participants with regard to the body scanning process and fit preference for a garment waistband, as well as gathering statistical analysis of quantitative dimensional data.

\subsection{Data collection}

\subsubsection{Ethics}

The scanning process had to be approved by MMU's ethics committee before the survey could take place. Prior to the scan day, subjects were given a written and visual account of the scan process and were informed they could withdraw at any time. This was verbally reiterated when participants arrived. Participants personal details were recorded on a password protected database, which allocated each person with a code for anonymity. Participants were required sign a consent form to be eligible for scanning. All forms were stored in a safe.

During the research field notes were transcribed both pre- and post-scanning so data concerning participants perceptions and reactions to recruitment, and the scanning process including the responses to the visuals were accurately documented.

\subsubsection{Measurement Equipment}

Two TC 2 scanners - the NX16 and KX16 models - were used to capture body surface data. The KX16 is portable and was used for the Nottingham survey. A customised measurement extraction profile file (MEP) was developed to extract specific height and girth measurements suitable for bodice development. This has been termed the MMU MEP. The waist definition within this MMU MEP has been developed by using the spinal curve to the lumber region and placing the landmark above the small of the back within a $4 \mathrm{~cm}$ tolerance. The same MMU MEP with the same waist definition was used for the entire sample prior to data analysis.

Participants removed all clothing except their own lingerie - as it was important they wore the intimate garments they would wear normally as this work would later on be developing block patterns to go over the lingerie. Subjects were shown how to stand in the scanner and were scanned three times to ensure a clear scan. Once dressed participants were asked if they wanted to see their scan on screen and if they wanted a printout.

\subsubsection{Waist position visual aid}

The visual aid (Fig 1 below) enabled subject participation in the landmark placement evaluation process; something that no other study had done before. The visual aid also facilitated the 'non-contact' nature of the measurement survey, as this was part of the attraction of the process to this demographic. As the aim of this research is to improve pattern development a skirt silhouette was used on the visual aid to articulate fit preference for a garment with a waistband.

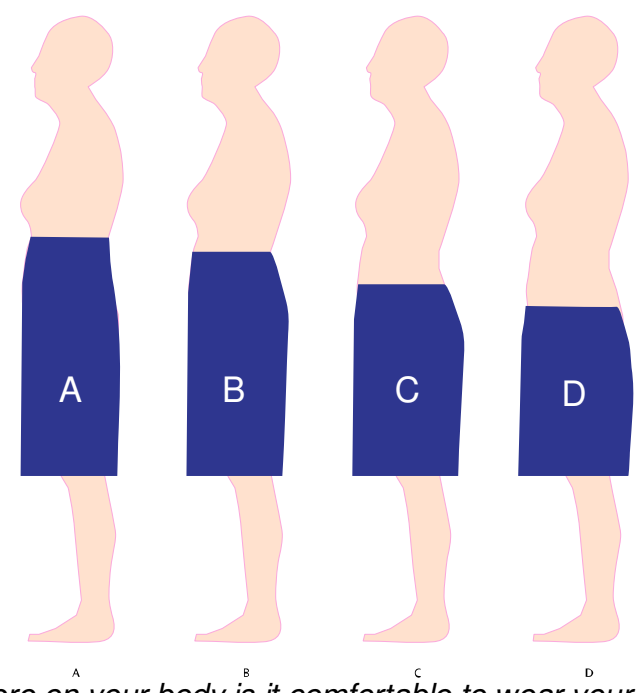

Fig. 1 Where on your body is it comfortable to wear your waistband? 
The visual aid was piloted using two MMU academics, both of whom fitted the recruitment criteria and had experience of the clothing product development process. Feedback from the pilot identified that the visual aid needed to be simple but not idealised. It was also determined that the visuals would depict the following definitions:

Table 1. Landmark definitions used in the visual aid

A. Using the spinal curves and placed above the small of the back with a 2.5" tolerance - [23]

B. Using the spinal curves and placed above the small of the back with a $4 \mathrm{~cm}$ tolerance provided within the TC[2] software. This definition was used in the MMU MEP

C. Using the spinal curves and placed at the small of the back - [22]

D. At the point of the top of the iliac crest - [43]

These definitions were selected because they have been used in previous research centered on anthropometric practice and they had enough scope to place the waist at visually different intervals along the torso.

\subsubsection{Developing the visual aid}

The aesthetic of the image needed careful consideration as the image's body shape and silhouette (Fig 1) needed to represent the demographic of the 55+ woman. Clothing retailers catering for this demographic typically uses images of younger women that are highly idealised and are aspirational in their presentation [44]. This study, however, has used a scan of a 61-year-old woman because the image, although not extreme in terms of corpulence or posture and simplified to protect anonymity, is of a 55+ woman. Her silhouette when compared to a younger women of comparable height and weight was such that her shoulders depict slight curvature, her breasts are positioned lower on the torso than that of the younger woman, and her abdomen protrudes more when viewed in profile. The above landmark definitions could also be applied to her body within the scanner software and this enabled the positioning of the blue skirt seen in the images below. The woman chosen also displayed a rectangle body shape, which, according to earlier studies [17], is a common shape for this demographic.

The images were shown in profile as the MMU MEP used the topology of the spine to place the waist landmarks. Women were asked to examine their own body and determine where they would wear a garment with a shaped waist area or waistband and select one of the four waist positions indicated by the blue skirt.

\subsection{Data evaluation and reduction}

\subsubsection{Evaluation of scan data using the chosen visuals}

The scans were evaluated on a case-by-case basis. Observations and modifications of the each scan were recorded in Excel. A grid of known dimensions was placed behind the point cloud image and posture was ascertained using Liechty et al's [45] posture guide for reference. Sources of shadows or occlusions were highlighted and their impact was documented. Measurements were then extracted using the MMU MEP as discussed above. Body segments were checked, starting from the chin point and moving downward, to determine correct landmark placement. Questionable landmark positions were compared against the definitions within the software. Automatic waist placement was compared against the visual aid waist position, as chosen by the participant. A different waist definition was manually applied using the software's 'modify point' facility and the centre back waist height landmark. Once the measurement level on the scan aligned with the participant chosen visual measurement extraction was taken again to assess the new measurement position and if any changes had impacted on neighboring landmarks and the ensuing measurements.

Body shape was also calculated for each subject using Lee et al's [46] calculations for female body shape categorization (FFIT) programmed into an Excel spreadsheet developed by three academics from the Department of Apparel at MMU. Body shape was recorded in a table alongside body asymmetry and excess fat deposition. The original and amended scans were saved and both were batch processed into an Excel sheet so that original and amended dimensions of waist height from 
floor could be compared. These dimensions were then subtracted from each other to provide a numerical value to indicate how much the landmark had moved in a vertical plane. For this study, this new dimension was termed the waist height difference (WHD). Body shape was also checked for correlation against the WHD to identify whether any particular body shape was prone to large WHD readings.

\subsubsection{Comparative visual analysis}

The results of the findings using the visual aids and the body shapes were collated and statistically evaluated. This was to determine if one particular waist position was repeatedly selected and to ascertain any correlation between body shape and waist position amendment within the sample set.

\section{Results and Discussion}

\subsection{Recruitment}

\subsubsection{South Manchester}

Although South Manchester has an approximate population of 36,000 females aged 55+ [47] the poster campaign only resulted to recruit 4 women out of 17 enquiries. Women who made initial contact were curious about the technology and were keen to engage verbally but expressed concerns about their body shape, poor fit, and lack of choice in terms of garment styling. This finding mirrors that of previous studies whereby verbal engagement with participants was relatively easy [26]. However, one of the reasons given for candidates not engaging further with the survey was a reluctance to travel. The NX16 model was used for scanning in Manchester and as this was a fixed structure, it meant that if people wanted to be scanned they needed to come to MMU. Past surveys that have used manual methods have the potential to be more mobile as the equipment is not as large and this may account for greater attendance. Candidates who enquired about the survey also expressed concerns surrounding modesty when they understood that they would have to remove their outer garments. This concern remained even after it was explained that they could watch a video of the scanning process via a web link to help allay fears. Some individuals also were confused that the 3D scanner was a piece of medical equipment.

Only seven members of staff from the university agreed to be scanned despite a university-wide email invitation which covered all academic and administrative staff. The reasons for not participating ranged from a fear of other colleagues seeing their scan data or a lack of interest in body measurement information, both of which were identified in the work of Lee et al [26]. Of the seven that were scanned four were known to the team suggesting that the familiarity with the scanning team allayed trust issues.

Purposive and snowball sampling was more successful in recruiting for the Manchester portion of the survey. Women known to the research team were invited to participate with positive results. As these women already knew the researchers there was already a relationship of trust. Participants appeared more content to experience scanning with their friends to support them.

\subsubsection{Nottingham}

Three clothing retailers were became involved in the recruitment process through contact with a member of the research team. Their involvement meant the event was promoted through radio, as well as using posters and email. The companies also provided participants with incentives to be scanned which could have contributed to willingness to participate.

\subsection{Observed participants scanning experience}

None of the participants from either Manchester or Nottingham complained about the experience of being inside the scanner booth. Fifteen women from the Manchester survey explicitly stated they viewed the scanning experience as an enjoyable event and would be engaging in further leisure activities such as dining out or shopping after they had been scanned. They came as small groups of friends and appeared excited about the experience with some asking if they could return for further scans to check body size and shape as part of an exercising of diet regime. The survey in Nottingham took 3 days and was busier than Manchester. This meant scanning had to quicker to ensure shorter waiting times to avoid people leaving. Greater numbers meant the team spent less time with individuals therefore on day two a mature woman - who had previously been through the scanning experience - joined the team to engage with the participants both pre and post-scanning to help answer any questions and provide each participant with the four visuals. 
There was a mixed reaction to viewing the scan image on the screen and it did provoke emotion. The majority of subjects were content to see their image although three women did not wish to view the image on screen and one woman asked for it to be removed from the viewing screen.

Waist landmark evaluation could have been undertaken between scanner operator and participant whilst looking at the scan image on screen but this could only happen if the participant was comfortable both looking at their image and having a third party scrutinise their image whilst they were present. However, Lee et al's [26] study suggested that some subjects were uncomfortable with this approach, a finding of this study too. When presented with the scan printout subjects frequently expressed displeasure at the image, stating that they appeared larger than they expected or lamenting that they were a more slender shape when they were younger. Participants appeared more focused on the scan image as comments were directed at the image rather than the dimensional data. However, two participants who worked as clothing practitioners complained that the waist measurements were larger than they expected with one participant measuring herself again with a tape measure to prove her point. Comments around the dimensions centered on the fact that often right and left sides of the body were different or a desire to understand which body measurements would be necessary for garment purchasing.

\subsection{Visual aid results}

80 individuals were given the opportunity to use the visual aid but only $52(65 \%)$ women responded by choosing one of the figures from the four provided. 28 participants were sent the visual aid via email to select an appropriate waistband position but only 9 responded. Therefore, the uptake of the use of the visual aid was most successful when offered in person during the scanning process.

It was observed that a number of participants performed a variety of self-evaluation actions prior to choosing a visual. Some women palpated their torso either sitting or standing, some viewed a current waistband placement providing comments on the comfort levels it provided, and some women bent to one side to locate their natural waist or talked to their companions about their thoughts when choosing a suitable visual. No one reported problems with deciding on where they would prefer to wear their waistband and all participants understood and were comfortable with the question.

No participants commented on the fact that the figures in the visual aid were depicted in profile although the participants viewed themselves either front on in a mirror or looking downward onto their body when locating the waist point. No one asked if the positions had particular landmark definitions. Indeed, landmarks were not mentioned by participants at all. Therefore, it could be concluded that subjects viewed the visual aid in an instinctive manner rather than in strictly objective manner.

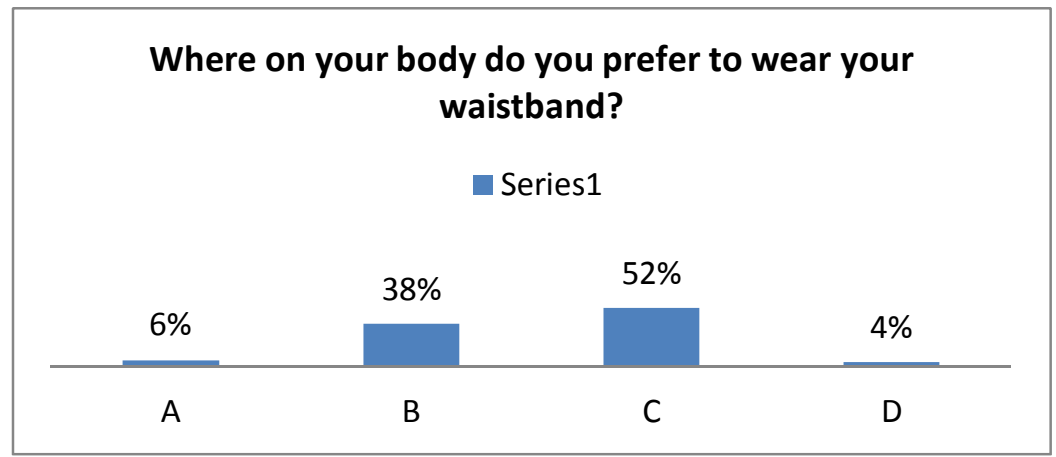

Fig. 2 Preferred waistband position

\subsubsection{Participant preferred waist positioning}

$52 \%$ of the sample identified visual C - around the small of the back - as the preferred position for their waistband. This could be a significant finding for pattern development as the small of the back could be used as a natural anchor point for a garment, depending on body shape, of course. This definition was used for the SizeUSA national anthropometric survey, a definition that according to Song \& Ashdown's [29] work was inappropriate for some individuals with a fuller figure.

$38 \%$ chose visual $B$ indicating they like to wear their waistbands up to $4 \mathrm{~cm}$ higher than their small of the back. This is the definition used by the MMU MEP and was in line with the UK's most recent national anthropometric survey. Therefore, it could be argued that this cluster of the sample would find UK garment fit appropriate, at least for waist height. 


\subsection{Scan data results}

\subsubsection{Most common age and body shape}

The sample ages ranged from 55-83 with $56 \%$ of the sample consisting of women mainly in their 60 s. The most common body shape was the rectangle (83\%), which concurs with earlier research [17]. Hourglass, bottom hourglass and triangle were represented in the sample so the survey was successful in capturing other body shapes (See Fig. 3 below).

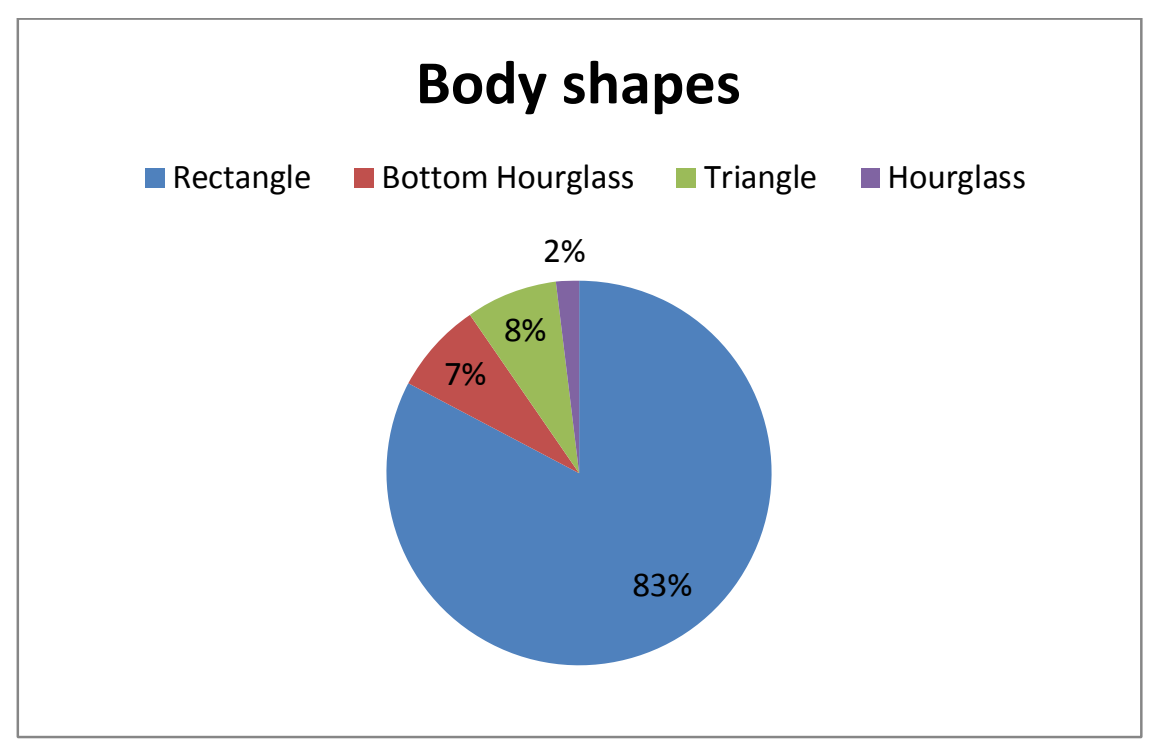

Fig. 3 Body shapes who participated

\subsubsection{Landmark modification}

$60 \%$ of the sample required the waist height landmark to be modified to correspond with their chosen visual. Of this $60 \%$, even though a number of subjects had selected visual $B-$ waist $=4 \mathrm{~cm}$ above the small of the back - and this corresponded to the MMU MEP, the landmark had to be manually adjusted to match the proportions on the visual they had chosen. The reason for waist height landmark modification in this instance was because the scanner software appeared to place the waist directly on or near the under-bust (Fig. 4), a result which has occurred in previous research [23].

What differs from previous research [23] is that the waist tolerance within landmark definition was much greater, at around 2.5", whereas the tolerance used in the MMU MEP was $4 \mathrm{~cm}$. The reduced tolerance made this process of moving the landmark to match the proportions of the visual aid more complex but this corresponded to the actual reduction evidenced between the small of the back and the bust in this demographic due to excess adipose fat in the abdomen and bust regions.

The body changes outlined in medical literature have clearly affected the waist positioning for this demographic, which provides a good rational for research of this nature. Nonetheless, regardless of body shape change, these women did not choose to have their waistband aligned to their under bust (see Fig. 5) and therefore the back waist landmark had to be moved to satisfy their requirements.
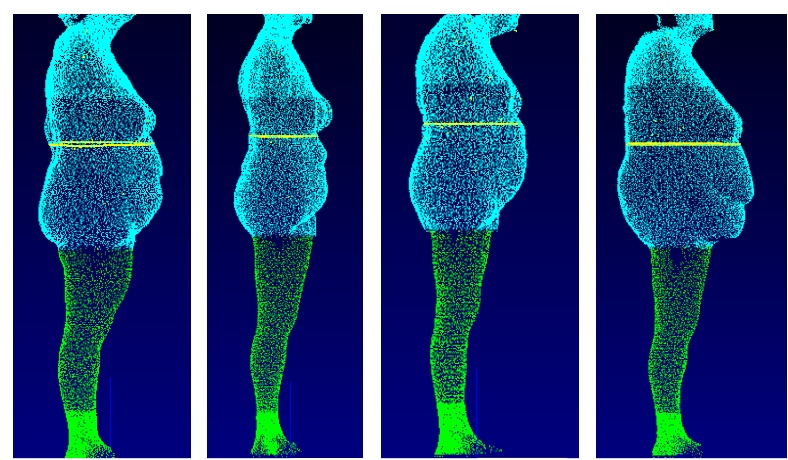

Fig. 4 waist positing lying on the under-bust measurement prior to waist amendment 


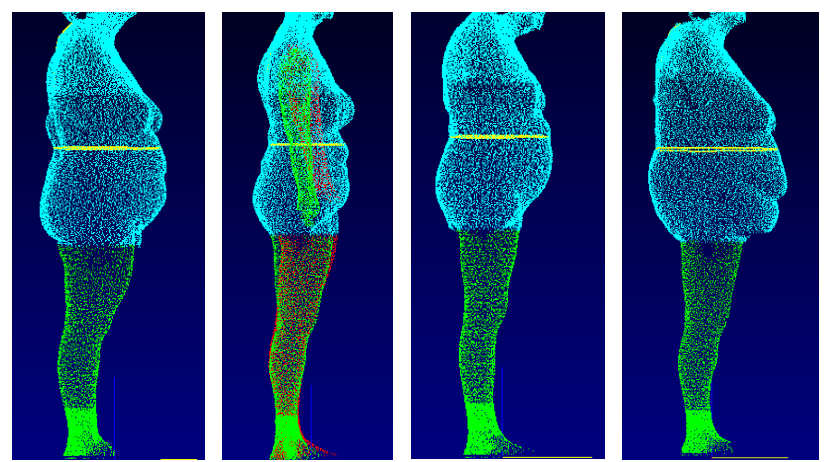

Fig. 5 waist positioning after amendment

\subsubsection{Body shape and landmark modification}

Fig. 6 below indicates that the rectangle shape had the greatest changes in waist height after modification. Three subjects who were rectangle shapes had chosen visuals which resulted large difference of waist height measurement between $-10.57 \mathrm{~cm}$ and $23.67 \mathrm{~cm}$. This occurred because the MMU MEP placed the waist at a significantly different position to the visual they chose. These 3 women either chose visual $A$ where the waist definition is placed high near the under bust and their small of the back was shallow and low on their body or the chose visual D, which is on the top of the iliac crest and the MMU MEP had placed their waist at the under bust. This meant the waist height landmark needed to be moved significantly if it were to match the visual they had chosen.

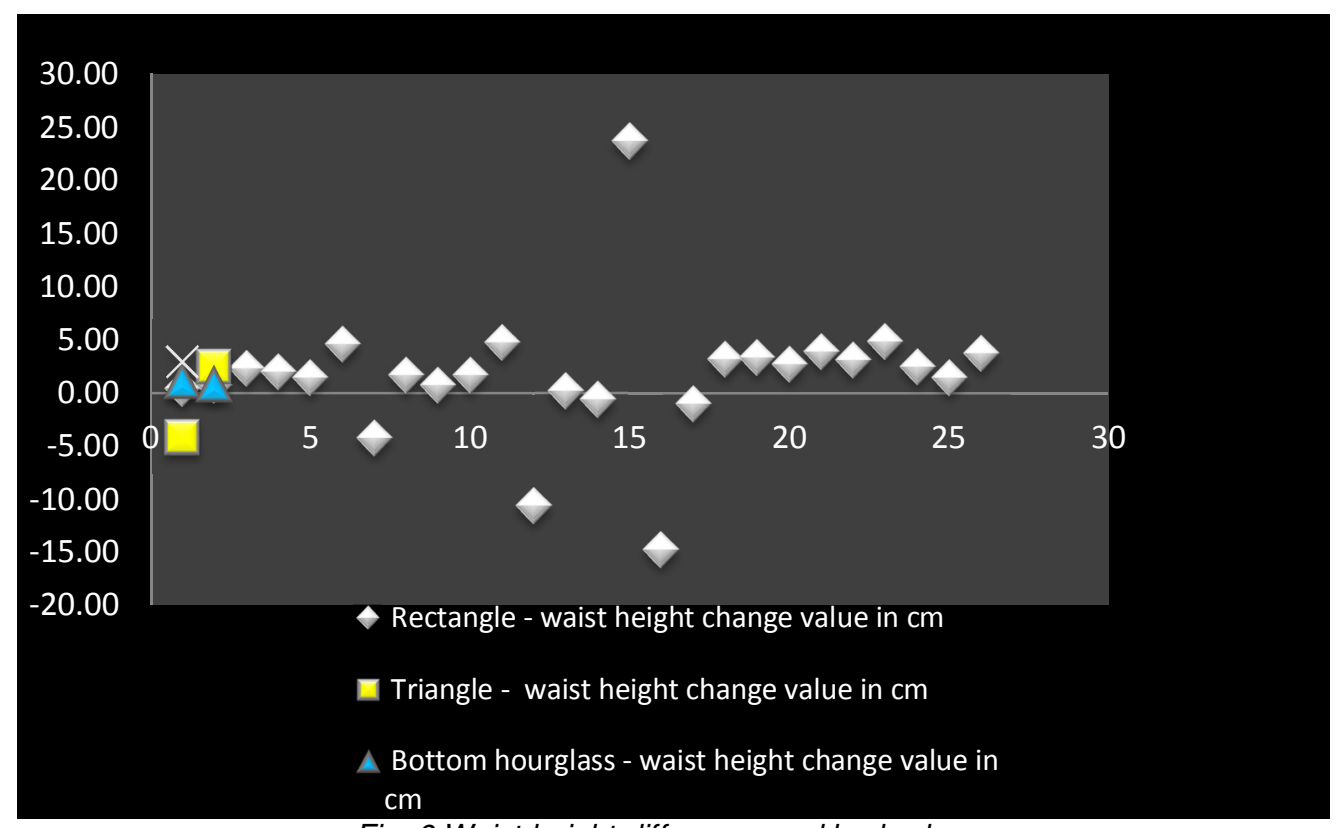

Fig. 6 Waist height difference and body shape

\section{Conclusion}

It is clear that current anthropometric practice using 3D body scanning is insufficient for this demographic as it does not allow for subject engagement as a means of confirming landmark positioning for people with non-standard morphologies.

The new process identified in this study has been specifically developed for application in garment product development. This process has not been discussed in any previous research and is therefore a new finding. This study concludes that to improve landmark placement accuracy a visual aid to the subject for evaluation in tandem with the practitioner evaluation would be a practice that is useful for common but difficult to locate landmarks, such as the waist.

When modifying a landmark position for the female 55+ demographic, the semantics and values of the definition used to direct the software was not successful for some subjects and the operator had to use their observational expertise to match the landmark positioning to the waistband positioning, as requested by the participant. 
This study acknowledges the practice entails familiarisation with anthropometric practice and knowledge of using the TC[2] interface therefore a level of training is needed. However, this new process does not require programming skills or detailed medical knowledge of the human body thereby making this a practice which could be readily adopted by clothing professionals.

\section{References}

[1] S. P. Ashdown, and E. O'Connell, "Comparison of test protocols for judging the fit of mature women's apparel", Clothing and Textile Research Journal, Vol. 24, issue 2, 2006, pp. 137-146

[2] A. Beazley, and T. Bond, "Computer-Aided Pattern Design \& Production Development, Oxford, Blackwell Publishing Ltd, 2003

[3] S. P. Ashdown, and L. Dunne, "A Study of Automated Custom Fit: Readiness of the Technology for the Apparel Industry", Clothing and Textiles Research Journal, Vol. 24, Issue 2, 2006, pp.121-136

[4] J, Croney, Anthropometry for Designers, London, Batsford Educational Limited, 1980

[5] C, D, Schewe, Marketing to our aging population: responding to physiological changes, The Journal of Consumer Marketing, Vol. 5, No. 3, 1988, pp. 61-73

[6] E, Bye, K, L, LaBat and M, R, Delong, Analysis of Body Measurement Systems for Apparel, Clothing and Textiles Research Journal, Vol. 24, No. 2, 2006, pp. 66-79

[7] S, Gill, and S, G, Hayes, Lower body functional ease requirements in the garment pattern, International Journal of Fashion Design, Technology and Education, 2011, ifirst - Available online: accessed 2011

[8] P, Kunick, Modern Sizing and Pattern Making For Women's and Children's Garments, London, Phillip Kunick Publications, 1984

[9] Bunka Fashion College, Garment Design Text Book 1: Fundamentals of garment design, Tokyo, Sunao Onuma, 2008

[10]M, Kouchi, and M, Mochimaru, Errors in Landmarking and the evaluation of the accuracy of traditional and 3D anthropometry, Applied Ergonomics, Vol. 42, No. 3, 2011, pp. 518-527

[11]J, D, Sorkin, D, C, Muller, and R, Andres, Longitudinal Change in Height of Men and Women: Implications for Interpretation of the Body Mass Index: The Baltimore Longitudinal Study of Aging, American Journal of Epidemiology, Vol. 150, No. 9, 1999, pp. 969-977

[12] R, Lindsey, D, M, Hart, C, Forrest and C, \& Baird, Prevention of spinal osteoporosis in oophorectomised women, The Lancet, Vol. 316, No. 8205, 1980, pp. 1151-1154

[13] S, P, Ashdown and H, Na, Comparison of 3-D Body Scan Data to Quantify Upper-Body Postural Variation in Older and Younger Women, Clothing and Textiles Research Journal, Vol. 26, No. 4, 2008, pp. 292-307

[14]C, J, Ley,B, Lees, and J, C, Stevenson, Sex- and menopause-associated changes in body-fat distribution, American Journal of Clinical Nutrition, Vol. 55, No. 5, 1992, pp. 950-954

[15] C, A, Patterson and J, Warden, Selected Body Measurements of Women Aged Sixty Five and Older, Clothing and Textiles Research Journal, Vol. 2, No 1, 1983, pp. 23-31

[16] E, M, Woodson and P, E, Horridge, Apparel Sizing as it Relates to Women Aged Sixty Five Plus, Clothing and Textiles Research Journal, Vol. 8, No. 4, 1990, pp. 7-13

[17]E, Goldsberry, S, Shim, and N, Reich, Women 55 years and older: Part I Current Body Measurements as Contrasted to the PS 42-70 Data, Clothing and Textiles Research Journal, Vol. 14, No. 2, 1996, pp. 108-120

[18] C, J, Salusso, J, J, Borkowski, N, Reich, and E, Goldsberry, An Alternative Approach to Sizing Apparel for Women 55 and Older, Clothing and Textiles Research Journal, Vol. 24, No. 2, 2006, pp. 96-111

[19]Y, Lee, and K, Nah, A study on the anthropometric change of Korean women's body shape for human centred product design, 2008 http://www.idemployee.id.tue.nl/g.w.m.rauterberg/conferences/cd_donotopen/adc/final_paper/629 .pdf , accessed 2011

[20] J, C, K, Wells, T, J, Cole, and P, Treleaven, Age-variability in Body Shape Associated With Excess Weight: The UK National Sizing Survey, Obesity, Vol. 16, 2008, pp. 435-441

[21]R, Suikerbuik, H, Tangelder, $H$, Daanen, \& A, Oudenhuijzen, Automatic Feature Detection in $3 D$ Human Body Scans http://www.cs.uu.nl/groups/AA/multimedia/publications/pdf/suikerbuik04.pdf, accessed 2010

[22] E, Kirchdoerfer, P, Treleaven, I, Douros and J, Bougourd, Proposed Human Body Measurement Standard, 2002, http://www0.cs.ucl.ac.uk/staff/p.treleaven/BodyMeasurements.pdf, accessed 2013 
[23]P, Devarajan and C, L, Istook, Validation of 'Female Figure Identification Technique (FFIT) for Apparel@ ' Software, Journal of Textile and Apparel Technology and Management, Vol. 1, No 2, 2004, pp. 1-23

[24] A, T, Wynne, M, A, Nelson, B, E, C, Nordin, Costo-Illiac Impingment Syndrome, The Journal of Bone and Joint Surgery, Vol.67-B, No. 1, 1985, pp. 124-125

[25] R, O'Brien, and W, C, Shelton, Women's Measurements for Garment and Pattern Construction (Public. No. 454, Department of Agriculture). Agriculture, U.S.D.o. Washington, Washington, DC: U.S. Government Printing Office. Miscellaneous Publication No. 454, 1941

[26]Y, A, Lee, M, L, Damhorst, M, S, Lee, J, M, Kozar, and P, Martin, , Older Women's Clothing Fit and Style Concerns and Their Attitudes Towards 3D Body Scanning, Clothing and Textiles Research Journal, Vol. 30, No. 2, 2012, pp. 102-118

[27] E, B, Rim, M, J, Stampfer, G, A, Colditz, C, G, Chute, L, B, Litin, and W, C, Willett, Validity of Self-Reported Waist and Hip Circumferences in Men and Women, Epidemiology, Vol. 1, No. 6, 1990, pp. 466-473

[28] R, S, Guerra, T, F, Amaral, E, A, Marques, J, Mota, and M, T, Restivo, , Anatomical Location for Waist Circumference Measurement in Older Adults; a Preliminary Study, Nutrición Hospitalaria, Vol. 27, No. 5, 2012, pp. 1554-1561

[29] H, K, Song, and S, P, Ashdown, Female Apparel Consumers' Understanding of Body Size and Shape: Relationship Among Body Measurements, Fit Satisfaction, and Body Cathexis, Clothing and Textiles Research Journal, Vol. 31, No. 3, 2013, pp. 143-156

[30]V, M, Haffenden, Knit to fit: applying technology to larger-sized women's body, 2009 http://eprints.brighton.ac.uk/7084/, accessed 2014

$[31] \mathrm{H}$, Joung, and N, J, Miller, , Factors of dress affecting self-esteem in older females, Journal of Fashion Marketing and Management, Vol.10, No. 4, 2006, pp. 466-478

[32] J, Nam, R, Hamlin, H, J, Gam, J, H, Kang, J, Kim, P, Kumphai, C, Starr, and L, Richards, The fashion-conscious behaviours of mature female consumers, International Journal of Consumer Studies, Vol. 31, No. 1, 2006, pp. 102-108

[33]M, A, V, Rocha, L, Hammond, and D, Hawkins, Age, gender and national factors in fashion consumption, Journal of Fashion Marketing and Management, Vol. 9, No. 4, 2005, pp. 380-390

[34]Z, Zhang, Y, C, Gong and H, Wu, Casual Wear product Attributes: A Chinese Perspective, Journal of Fashion Marketing and Management, Vol.6, No. 1, 2002, pp. 53-62

[35]B, Newcombe, and C, Istook, A Case for the Revision of Sizing Standards, The Journal of Textile and Apparel Technology, Vol. 4, No. 1, 2004, pp. 1-6

[36]M, L, Richards, The Clothing Preferences and Problems of Elderly Female Consumers, Gerontologist, Vol. 21, No. 3, 1981, pp. 263-267

[37] H, A, M, Daanen, and S, Hong, Made-to-measure pattern development based on 3D whole body scans, International Journal of Clothing Science and Technology, 20 (1), 2008, pp. 15-25

[38] R, Maisel, and C, Hodges-Persell, How Sampling Works, California, Pine Forge Press, 1996

[39] S, Saratakos, Social Research, $4^{\text {th }}$ Edition, Basingstoke, Palgrave MacMillan, 2013

[40] M, B, Miles, and A, M, Hubermann, Qualitative Data Analysis: An Expanded Sourcebook, $2^{\text {nd }}$ Edition, USA, Sage Publications, 1993

[41]BSI (2010), BS EN ISO 20685:2010, 3D scanning methodologies for internationally compatible anthropometric databases, British Standards Institute

[42] J, W, Creswell and V, L, Plano-Clark, Designing and Conducting Mixed Methods Research, $2^{\text {nd }}$ Edition, USA, Sage Publications, 2011

[43] J, Wang, J, C, Thornton, S, Bari, B, Williamson, D, Gallagher, S, B, Heymsfield, M, Horlick, D, Kotler, B, Laferrere, L, Mayer, F, X, Pi-Sunyer, and R, N, Pierson Jr, Comparisons of Waist Circumferences measured at 4 sites, The American Journal of Clinical Nutrition, Vol. 77, 2003, pp. 379-384

[44]J, M, Kozar and M, L, Damhorst, Comparison of the Ideal and Real Body as Women Age: Relationships to Age Identity, Body Satisfaction and Importance, and Attention to Models in Advertising, Clothing and Textiles Research Journal, Vol. 27 No. 3, 2009, pp. 197-210

[45] E, L, Liechty, D, N, Pottberg-Steineckert, and J, A, Rasband, Fitting and Pattern Alteration, $2^{\text {nd }}$ Edition, New York, Fairchild Books, 2009

[46] J, Y, Lee, C, L, Istook, Y, J, Nam, and S, M, Park, Comparison of Body Shape between USA and Korean Women, International Journal of Clothing Science and Technology, Vol. 19, No. 5, 2007, pp. 374-391

[47] Public Intelligence Population

Publications,http://www.manchester.gov.uk/downloads/download/4220/public_intelligence_popula tion_publications, accessed 2014 